\title{
Renovascular hypertension masquerading as nephrotic proteinuria in an 10-month-old girl — analysis of errors in diagnostic approach
}

\author{
Łukasz Obrycki, Anna Niemirska, Mieczyslaw Litwin \\ Department of Nephrology, Kidney Transplantation and Hypertension, The Children's Memorial Health Institute, Warsaw, Poland
}

\begin{abstract}
A 10-month-old girl with nephrotic proteinuria, hypercholesterolaemia, hypoalbuminaemia, hyponatraemia, hypokalaemia, hypocalcaemia, metabolic alkalosis, normal auscultatory blood pressure on multiple occasions, and an enlarged left kidney on ultrasound, was referred to hospital. A left-kidney biopsy showed normal glomeruli with mild mesangial proliferation and the thickening of arteriolar walls. Glucocorticoid therapy was started. A further course was complicated by vomiting, irritation, pulsatile fontanel, and an enlarged ventricular system on ultrasound. A ventriculoperitoneal shunt was inserted but, during anaesthesia, repeated automated, oscillometric measurements revealed blood pressure of $220 / 120 \mathrm{mmHg}$. Intravenous antihypertensive treatment lowered the blood pressure to $110-120 / 70 \mathrm{mmHg}$, with a decrease in proteinuria. A CT abdominal angiography revealed left-renal-artery stenosis, a reduced right kidney and an enlarged left kidney, with foci of a post-infarct area in the lower pole. The patient was referred to a tertiary-care hospital. Renal scintigraphy showed that the right-kidney function had decreased to $6 \%$. A right nephrectomy was performed but, due to persistent arterial hypertension, oral antihypertensive treatment was intensified. Microscopic analysis of the removed right kidney revealed relatively normal glomeruli and fibromuscular dysplasia in the right-renal-artery specimen. Oral antihypertensive therapy based on an angiotensin-converting enzyme inhibitor and a calcium-channel blocker continued after surgery allowed the attaining of normotension, the gradual normalisation of proteinuria and metabolic abnormalities, and an improvement in the left-kidney function. This case shows that auscultatory blood-pressure measurements in small children and incorrect interpretation of the obtained test results can lead to the erroneous misdiagnosis of hypertensive crisis, with dramatic consequences. Key words: hypertension, infants, blood pressure measurement, renovascular hypertension, nephrotic syndrome
\end{abstract}

Arterial Hypertens. 2018, vol. 22, no. 3, pages: 143-149

DOI: 10.5603/AH.a2018.0010

\section{Introduction}

Renovascular hypertension (RVH) is one of the major causes of severe hypertension in children and adolescents. The main cause of RVH in childhood is fibromuscular dysplasia (FMD), but in $20-40 \%$ of cases RVH is the consequence of other diseases (syndromic RVH), including type-1 neurofibromatosis (> 15\% of all cases of RVH) [1-3]. RVH can also be caused by congenital or acquired stenoses of the renal artery, accessory renal arteries and/or segmental arteries $[4,5]$. Clinical findings are non-specific, and are the consequences of both metabolic abnormalities caused by the activation of the renin-angiotensin-aldosterone system (RAAS), hypertensive injury of kidney with patent renal artery, and direct hypertensive cardiovascular damage caused by high blood

Address for correspondence: Lek. Łukasz Obrycki

Klinika Nefrologii, Transplantacji Nerek i Nadciśnienia Tętniczego, Instytut „Pomnik-Centrum Zdrowia Dziecka”

Aleja Dzieci Polskich 20, 04-730 Warszawa

tel.: 228151540

e-mail: I.obrycki@ipczd.pl 
pressure. Thus, patients with RVH can present wide spectrum of clinical signs and symptoms from headache to life-threatening signs of the breakdown of autoregulatory mechanisms with brain oedema, glomerular hyperfiltration, heart failure or stroke. The activation of the RAAS leads to secondary hyperaldosteronism with hypokalaemia and metabolic alkalosis. The exposure of the kidneys with normal artery to high systemic blood pressure leads to glomerular hyperfiltration, causing albuminuria and pressure natriuresis. Thus, the first step in the diagnosis of $\mathrm{RVH}$ is the detection of an elevated blood pressure and proper interpretation of laboratory and radiological findings because the clinical symptoms are non-specific. According to the guidelines, auscultatory measurement is the referential method confirming the diagnosis of $\mathrm{AH}[6]$. However, auscultatory measurement has its own limitations, which should be considered [7]. First, auscultatory blood-pressure measurement is difficult to take in neonates and small children. Second, one can erroneously treat diastolic blood pressure as systolic blood pressure. This is why recent guidelines recommend the use of validated automatic oscillometric measurements in small children $[8,9]$.

In most cases $\mathrm{RVH}$ is a treatable cause of $\mathrm{AH}$. However, even the successful revascularisation or nephrectomy of an ischaemic kidney might not lead to normotension and full recovery. One of the causes is persistent hypertensive injury to the contralateral kidney with patent renal artery and exposed to high blood pressure before the revascularisation/nephrectomy of an ischaemic kidney.

We present the case of RVH "masquerading" as nephrotic syndrome in an infant in whom delayed diagnosis, caused by an improper method of blood-pressure measurement and erroneous interpretation of laboratory and radiological data, led to severe complications.

\section{Case report}

A 10-month-old girl, previously healthy, with a family history of Hodgkin's lymphoma in the father, was admitted to the Department of Paediatric Nephrology of the Regional Hospital because of nephrotic range proteinuria and erythrocyturia, found during an investigation of the failure to thrive, poor appetite and irritability. No abnormalities, except irritability, were found on physical examination. Repeated multiple auscultatory blood-pressure measurements showed systolic blood-pressure values in the range of 115 to $120 \mathrm{mmHg}$. Laboratory blood tests revealed mild hyponatraemia, hypokalaemia and metabolic alkalosis, hypocalcaemia with normal ionised calcium concentrations, slight hypercholesterolaemia, and hypoalbuminaemia (Table I). The 24-hour urinary-protein excretion was $166 \mathrm{mg} / \mathrm{kg}$. The concentration of $\mathrm{C} 3$ and $\mathrm{C} 4$ complement components was normal, and no antinuclear or antineutrophil cytoplasmic antibodies were found. HBV, HCV, EBV, $\mathrm{CMV}$ and rubella tests were negative. Ultrasonography revealed increased echogenicity in the renal cortex, and decreased corticomedullary differentiation in both kidneys. The left kidney was described as enlarged to $8 \mathrm{~cm}$, the right-kidney length was $5 \mathrm{~cm}$, and no enlargement of the pelvicalyceal system was found. In the dynamic renal DTPA scintigraphy, a slightly larger left kidney was described, with $56 \%$ of filtration function, and the right kidney was slightly smaller, accounting for $44 \%$ of the relative

Table I. Changes of biochemical markers measured at first admission and during follow-up

\begin{tabular}{|l|c|c|c|}
\hline & $\begin{array}{c}\text { Laboratory test value at the } \\
\text { beginning of the symptoms }\end{array}$ & $\begin{array}{c}\text { Laboratory test value during blood } \\
\text { pressure lowering (6 weeks after } \\
\text { nephrectomy) }\end{array}$ & $\begin{array}{c}\text { Laboratory test value during blood } \\
\text { pressure lowering (1.5 years after } \\
\text { nephrectomy) }\end{array}$ \\
\hline Serum creatinine $[\mathrm{mg} / \mathrm{dl}]$ & 0.22 & 0.40 & $(-39)$ \\
\hline $\begin{array}{l}\text { Protein concentrations in spot } \\
\text { urine }[\mathrm{mg} / \mathrm{dll}]\end{array}$ & 1590 & 30 & 138 \\
\hline Serum sodium $[\mathrm{mmol} / \mathrm{l}]$ & 127 & 137 & 5.2 \\
\hline Serum potassium $[\mathrm{mmol} / \mathrm{l}]$ & 2.91 & 4.7 & 2.61 \\
\hline Serum calcium $[\mathrm{mmol} / \mathrm{l}]$ & 2.20 & 2.55 & 22.4 \\
\hline Venous $\mathrm{HCO}[\mathrm{mmol} / \mathrm{m}]$ & 34.5 & 22.1 & -1.6 \\
\hline Base excess $[\mathrm{mmol} / \mathrm{l}]$ & 14.1 & -2.5 & 43.1 \\
\hline Serum albumin $[\mathrm{g} / \mathrm{l}]$ & 29.2 & 41.4 & 163 \\
\hline Total cholesterol $[\mathrm{mg} / \mathrm{dl}]$ & 258 & 145 & \\
\hline
\end{tabular}




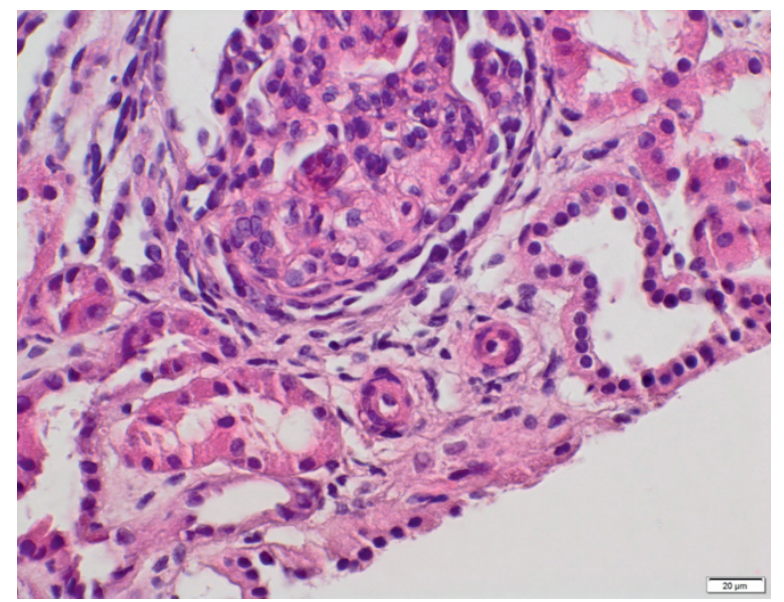

Figure 1. Renal biopsy. Expanded mesangium ( $>3$ cells in one loop), arteriolar changes typical for hypertensive nephropathy (thickening of the arteriolar wall, reduced arteriolar lumen)

kidney function. Contrast-enhanced computed tomography (CT) (without angiography) found an enlarged left kidney $(79 \mathrm{~mm})$, with retained corticomedullary differentiation, and reduced but preserved contrast enhancement with discrete, uneven hyperintensity of the kidney parenchyma, seen in the delayed phase. The right kidney was described as normal. Due to the clinical and biochemical features of the nephrotic syndrome, the suspicion of cancer (Hodgkin lymphoma in a father, enlargement of one kidney, early age of the patient), and the enlargement of left kidney with the relatively normal size of the right kidney, a biopsy of the left kidney was done. Microscopic examination revealed mesangial proliferation with an increased mesangial matrix, and the thickening of the arteriolar wall, with a decrease in the lumen diameter (Figure 1). Nephrotic syndrome was diagnosed, and corticosteroid treatment was started. Multiple blood-pressure values obtained with the auscultatory method were reported in the range of $115-120 \mathrm{mmHg}$, and enalapril with amlodipine were added. A few days after the start of the prednisone treatment, the patient presented with vomiting and somnolence. Tense and pulsating frontal fontanelle was found during the examination. In laboratory tests, the inflammatory markers were negative, and hyponatraemia $(125 \mathrm{mmol} / \mathrm{l})$ and metabolic alkalosis were noted. In transcranial ultrasound an enlarged ventricular system was found. CT scan of the head confirmed the enlargement of the brain ventricles (the lateral ventricles in the frontal horns up to 22 $\mathrm{mm}$ wide, the third chamber width $14 \mathrm{~mm}$ and the fourth chamber width $12 \mathrm{~mm}$ ). Low-density areas around the corners of the frontal horns of the lateral ventricles were found, indicating transependymal cerebrospinal fluid flow. The cerebromedullary cistern was also expanded. Ophthalmic examination found optic nerve oedema. The child was qualified for surgery. The blood pressure measured by the auscultatory technique before the surgery was reported as normal. Ventriculoperitoneal shunting was performed. During the surgery, the blood pressure measured with an automated oscillometric device was found to be $220 / 120 \mathrm{mmHg}$. Hypertensive crisis was diagnosed, and an intravenous antihypertensive therapy with urapidil and hydralazine was started, with a subsequent decrease in systolic blood pressure during the next 24-36 hours to 120$-130 \mathrm{~mm} \mathrm{Hg}$, as measured with an automatic oscillometric device. Prednisone was stopped. Total protein and serum albumin concentrations were decreased, and the daily protein excretion was $128 \mathrm{mg} / \mathrm{kg} / 24 \mathrm{~h}$. The laboratory tests were repeated and computed tomography angiography (angioCT) performed. An over-50\% decrease in the lumen diameter of the right proximal renal artery, around $3 \mathrm{~mm}$ in length, was found. In the distal segment of the right renal artery and its branches no stenosis was observed. In the left kidney, 2 renal arteries were found, without any stenosis described. At the lower-left-kidney pole, a discrete hypodense area was observed, absent in the previous CT (Figure 2). The girl was admitted to the Department of Nephrology Kidney Transplantation and Hypertension in the Children's Memorial Health Institute.

Blood pressure on admission was up to 130 $-172 / 105 \mathrm{mmHg}$ despite the combined intravenous treatment with urapidil and hydralazine. The continuous infusion of labetalol and hydralazine was started, and oral antihypertensive drugs were

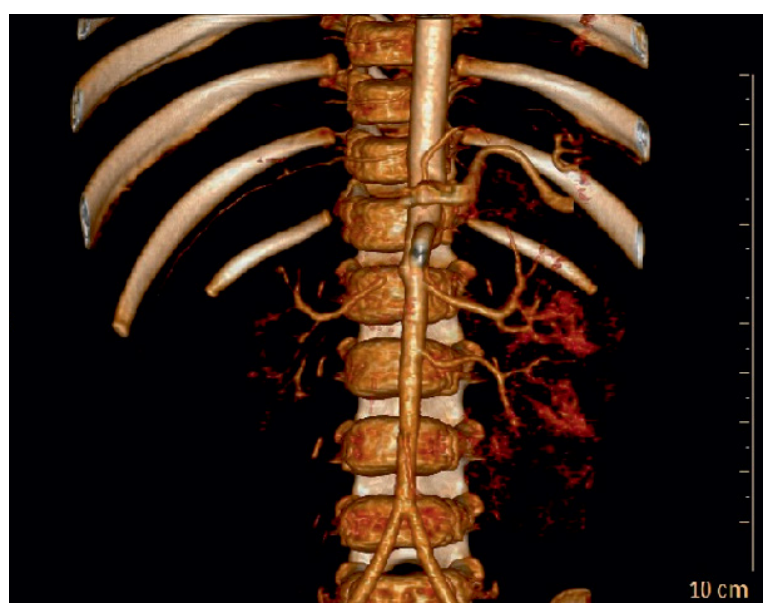

Figure 2. Computed angiotomography. The narrowing of the right renal artery (in the right proximal renal artery, at the section of about $3 \mathrm{~mm}$, a narrowing of the vessel to at least $50 \%$ of the diameter of the lumen) 


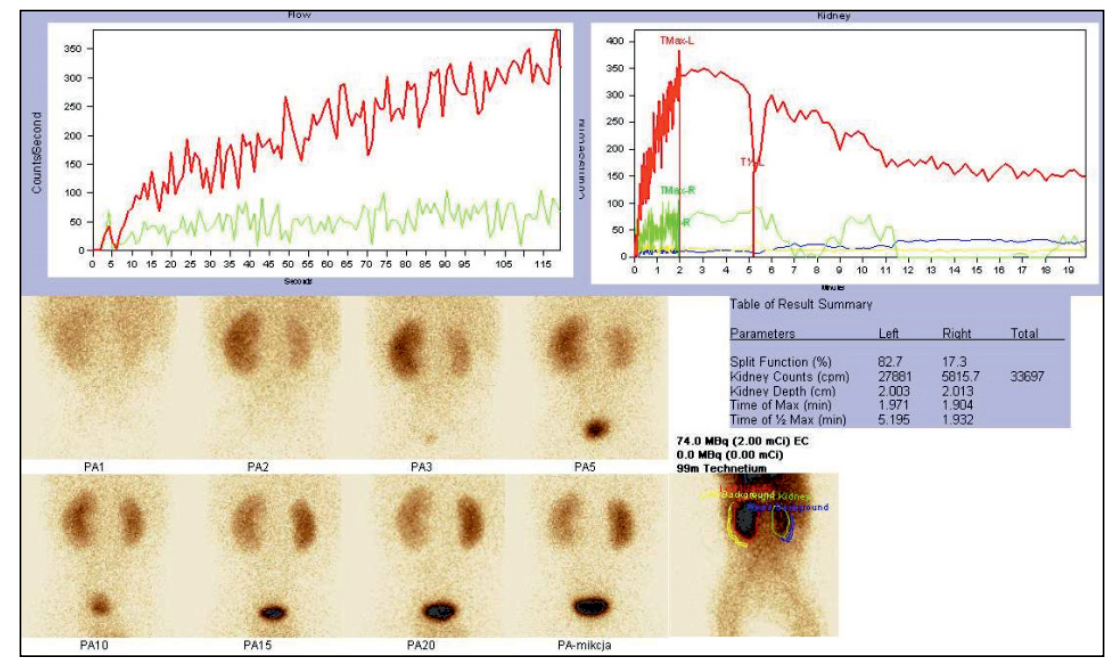

Figure 3. Dynamic renal scintigraphy with ethylenedicysteine at admission. Small right kidney, with significantly impaired secretory function, prolonged retention of the tracer and difficult to assess excretion. Left kidney with efficient secretory and excretory function. The right kidney filtration split fraction is $17 \%$

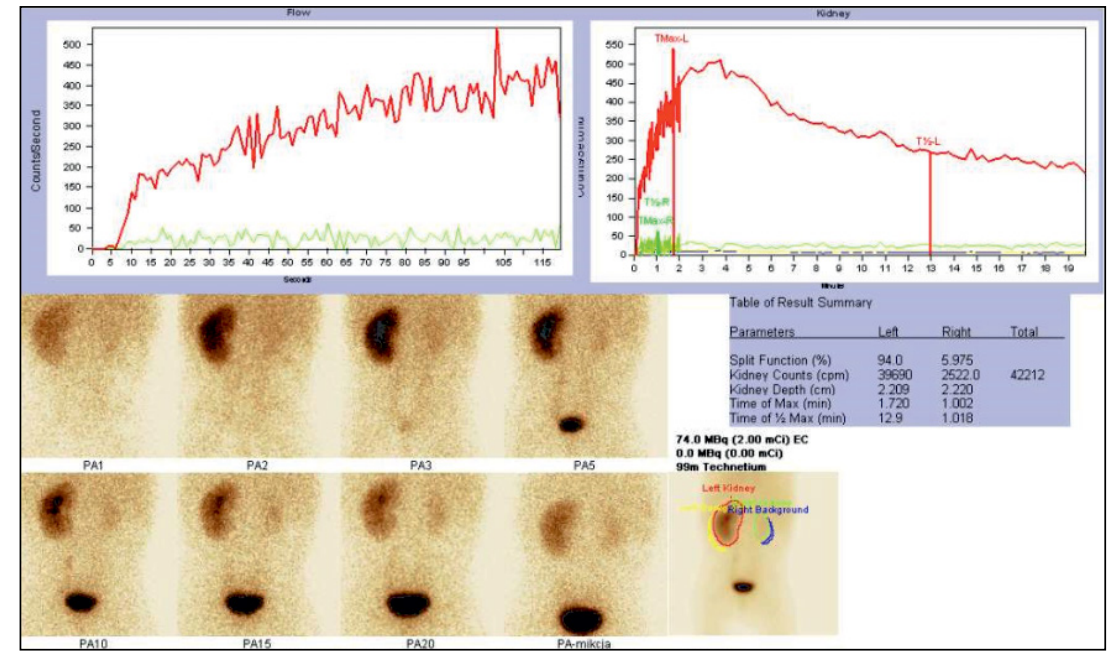

Figure 4. Dynamic renal scintigraphy with ethylenedicysteine after 7 days of antihypertensive treatment (BP 100/60 mmHg). The right kidney filtration split fraction decreased to $6 \%$

added. Doppler abdominal ultrasonography revealed a smaller right kidney (length $55 \mathrm{~mm}$ ) with post-stenotic spectrum of the blood flow in the segmental arteries (prolonged acceleration time). The Doppler-flow parameters of the left kidney (length $77 \mathrm{~mm}$ ) were normal. Dynamic renal scintigraphy revealed small right-kidney functioning with $17 \%$ of split filtration fraction and, with significantly prolonged cortical retention of the radiotracer (Figure 3). In echocardiography and fundoscopic examination no abnormality was found. The protein in spot urine was $373 \mathrm{mg} / \mathrm{dl}$, without erythrocyturia and leucocyturia, and a slight hypoalbuminaemia $(34.7 \mathrm{~g} / \mathrm{l})$ was observed. GFR was normal. In the control, dynamic kidney scintigraphy was performed when blood pressure decreased to normal values, and the right kidney filtration split fraction decreased to 6\% (Figure 4).

Because of the lack of post-stenotic dilatation, and low kidney functioning, a right nephrectomy was done. After nephrectomy, the blood pressure slightly decreased, but the patient still needed antihypertensive therapy (amlodipine, propranolol, doxazosin, captopril and spironolactone). After lowering blood pressure to mean $100 / 60 \mathrm{mmHg}$ values, proteinuria decreased to $82 \mathrm{mg} / \mathrm{dl}$ in spot urine. At discharge, blood-pressure values were in the range of 100-90/ $168 \mathrm{mmHg}$, and protein concentration in the spot urine decreased to $30 \mathrm{mg} / \mathrm{dl}$. Six months after nephrectomy, BP in home oscillometric measurements was 
in the range of $90 / 50-100 / 60 \mathrm{mmHg}$, the protein concentration in the urine was $10 \mathrm{mg} / \mathrm{dl}$. The number of antihypertensive drugs was gradually reduced to three (propranolol, amlodipine and captopril), and then to two (captopril and amlodipine). After one year, $\mathrm{BP}$ was $85 / 60 \mathrm{mmHg}$, and no proteinuria was detected in spot urine. The serum creatinine was $0.39 \mathrm{mg} / \mathrm{dl}\left(\mathrm{eGFR} 94 \mathrm{ml} / \mathrm{min} / 1.73 \mathrm{~m}^{2}\right.$ ).

\section{Discussion}

Clinical signs and symptoms of RVH are non-specific, and only blood pressure measurement may allow to start diagnostic procedures to find the exact cause of arterial hypertension. Our report presents the consequences of an erroneous diagnosis in an 10-monthold girl who presented with nephrotic proteinuria that was diagnosed as a nephrotic syndrome but, in fact, indicated hypertensive crisis lasting for several weeks. The girl initially presented symptoms of a nephrotic syndrome (nephrotic proteinuria, hypercholesterolaemia, decreased serum albumin level), caused by hypertensive nephropathy of kidney with patent renal artery and exposed to extremely high systemic blood pressure. Patient's blood pressure was first measured using an auscultatory sphygmomanometry method. Numerous recordings of blood pressure measured with this method were reported as normal or slightly elevated, which was interpreted as associated with irritability and as a symptom secondary to a renal disease. However, auscultatory measurements are especially difficult in infants and small children. It is highly likely that during such measurements there might be interpretational errors, where diastolic blood pressure can be interpreted as systolic blood pressure. In contrast, in oscillometric devices, the pressure in the cuff adjusts to the mean arterial pressure, and the cuff is pumped up, and in the case of atypical values the device accommodates the pressure in the cuff even to very high levels. This might have been the case during the possibly first oscillometric measurement which was performed during neurosurgery. Although auscultatory measurements are regarded as a confirmatory method for the diagnosis of an elevated blood pressure in children, one must consider that in infants and small children oscillometric blood-pressure measurements are related to the lower prevalence of the white-coat effect, and are investigator-independent. Thus, according to the recently published American Academy of Paediatrics guidelines, blood pressure in neonates and infants should be measured with a validated oscillometric device [6]. In the described case, the oscillometric blood pressure measurement led to the proper diagnosis of arterial hypertension.

The clinical and laboratory symptoms observed in the described case were consequences of high blood pressure, and the activation of the renin-angiotensin-aldosterone system (RAAS). These include metabolic disturbances caused by secondary aldosteronism, i.e. hypokalaemia and metabolic alkalosis. Non-specific neurological symptoms due to brain oedema, such as irritability, somnolescence or seizures might be present. This complication was expressed as non-specific neurological symptoms, bulging fontanelle, and optic nerve oedema. Proteinuria, sometimes massive, is caused by glomerular hypertension, and is observed in patients with a unilateral renovascular disease. Pressure natriuresis can even lead to hyponatraemia presenting as a hyponatremic-hypertensive syndrome, as it was also observed in the described case. The next point was the false interpretation of the differences in kidneys length. Because the left kidney was described as being of normal length, and the right kidney was enlarged, with disturbed cortico-medullary differentiation, the suspicion of a possible lymphoma was raised, and a renal biopsy was performed. However, careful secondary analysis revealed a compensatory hypertrophy of the right kidney, with patent renal artery and foci of ischaemia, probably due to malignant hypertensive arteriopathy. Such a view was supported by microscopic findings.

Hypertensive nephropathy $(\mathrm{HN})$ is very rarely presented in children. The basis of HN is hypertensive injury to small arterioles, with hypertrophy of the media. In contrast to benign hypertensive arteriopathy, malignant hypertensive arteriopathy is characterised by fibrinoid necrosis of the arteriolar wall. In the discussed case, a renal biopsy revealed significant hypertrophy of the arteriolar wall, with narrowing of the lumen. The glomeruli were of normal size, and mesangial proliferation was found. However, despite the well-known association between hypertension and a chronic kidney disease, the underlying mechanism remains unclear. There are two proposed mechanisms of HN pathophysiology. Both centre on how the glomerulus is affected, with one theory identifying glomerular ischaemia as the main contributor to $\mathrm{HN}$, and the other identifying glomerular hypertension and glomerular hyperfiltration as the centre of the HN's pathogenesis [10]. Mesangial proliferation found in the kidney biopsy in the described case seems to be a non-specific reaction to glomerular injury, caused by hypertension and hyperfiltration. The fact that proteinuria was associated with pressure diuresis 
Table II. Changes in proteinuria (spot urine) [mg/dl]

\begin{tabular}{|l|c|c|}
\hline $\begin{array}{l}\text { Time from the diagnosis of arterial hypertension } \\
\text { and start of antihypertensive treatment }\end{array}$ & $\begin{array}{c}\text { Blood pressure values } \\
\text { in office measurements [mmHg] }\end{array}$ & $\begin{array}{c}\text { Protein concentrations } \\
\text { in spot urine [mg/dl] }\end{array}$ \\
\hline${ }^{\prime \prime}$ & max. 220/120 & 1590 \\
\hline 2 weeks & $120 / 70-140 / 90$ & 107 \\
\hline 3 weeks & $127 / 78-172 / 105$ & 373 \\
\hline 4 weeks & $110 / 64-139 / 73$ & 89 \\
\hline 5 weeks & $116 / 75-122 / 73$ & 72 \\
\hline 6 weeks & $100 / 60-110 / 68$ & 30 \\
\hline 8 weeks & $90 / 50-100 / 60$ & 10 \\
\hline 24 weeks & $100 / 60$ & 15 \\
\hline 1.5 year & $<90 / 60$ & $1-)$ \\
\hline
\end{tabular}

and hyperfiltration is further evidenced by gradual decrease and normalization after BP normalisation (Table II). The finding of increased thickness of arteriolar walls without any signs of fibrinoid necrosis does not fulfil the criteria of malignant hypertensive arteriolopathy and may be interpreted as a reaction to long-term lasting hypertension. Similarly, mesangial proliferation may be the first step to the development of glomerular sclerosis.

A right-kidney nephrectomy led to the lowering of blood pressure, but the patient still required antihypertensive treatment. Full normalization of blood pressure was achieved only after a few weeks. The lowering of blood pressure was accompanied by a decrease in proteinuria, and after 8 weeks proteinuria normalized. However, the patient still required two antihypertensive drugs (captopril and amlodipine). It was reported in numerous studies that the long-term prognosis and kidney functioning were normal in children with $\mathrm{RVH}$, treated with angioplasty or unilateral nephrectomy $[5,11,12]$. However, the described case presented with an already-developed significant arteriolar and glomerular injury. Thus, although clinical and laboratory remission of hypertensive nephropathy was achieved, it is not clear how microscopic changes found on the renal biopsy will evolve in the future. We decided to use an angiotensin-converting enzyme inhibitor and a calcium channel blocker because these drugs can lead to the regression of hypertensive target-organ damage and, in the case of the blockers of renin-angiotensin system, have renoprotective properties.

The main lesson learnt from our case is that proper blood pressure measurement and its interpretation are the bases for the proper diagnosis of arterial hypertension. Auscultatory sphygmomanometric measurements, despite being regarded as the gold standard, are associated with numerous investigator-dependent biases. These biases are especially significant in the case of neonates and small children. On the contrary, automated oscillometric measurements are investigator-independent, and are now recommended for blood pressure measurements in the youngest children. The other lesson learnt from the described case is the need for the proper interpretation of clinical and laboratory findings which would allow the avoidance of unnecessary and dangerous treatments. Third, although the renal biopsy of enlarged kidney was not necessary in our opinion, it showed the consequences of long-lasting hypertensive crisis on the renal circulation and glomerular structure. These findings provide an argument for prolonged antihypertensive and renoprotective therapy, even after the normalization of blood pressure.

\section{References}

1. Olin JW, Gornik HL, Bacharach JM, et al. Fibromuscular dysplasia: state of the science and critical unanswered questions: a scientific statement from the American Heart Association. Circulation. 2014; 129(9): 1048-1078, doi: 10.1161/01.cir.0000442577.96802.8c, indexed in Pubmed: 24548843.

2. Green R, Gu X, Kline-Rogers E, et al. Differences between the pediatric and adult presentation of fibromuscular dysplasia: results from the US Registry. Pediatr Nephrol. 2016; 31(4): 641-650, doi: 10.1007/s00467-015-3234-z, indexed in Pubmed: 26525198.

3. Slovut DP, Olin JW, Slovut DP, et al. Fibromuscular dysplasia. N Engl J Med. 2004; 350(18): 1862-1871, doi: 10.1056/NEJMra032393, indexed in Pubmed: 15115832.

4. Anderson WP, Kett MM, Stevenson KM, et al. Renovascular hypertension: structural changes in the renal vasculature. Hypertension. 2000; 36(4): 648-652, doi: 10.1161/01.hyp.36.4.648.

5. Antoniewicz J, Litwin M, Pedich M, et al. Diagnosis and treatment of renovascular hypertension in children and adolescents - single center experience with 87 patients. J Hypertens. 2007; 25: 332 .

6. Flynn J, Kaelber D, Baker-Smith C, et al. Clinical Practice Guideline for Screening and Management of High Blood Pressure in Children and Adolescents. Pediatrics. 2017; 140(3): e20171904, doi: 10.1542/ peds.2017-1904, indexed in Pubmed: 29192011. 
7. Chio SS, Urbina EM, Lapointe J, et al. Korotkoff sound versus oscillometric cuff sphygmomanometers: comparison between auscultatory and DynaPulse blood pressure measurements. J Am Soc Hypertens. 2011; 5(1): 12-20, doi: 10.1016/j.jash.2010.10.005, indexed in Pubmed: 21269907.

8. Dionne JM, Abitbol CL, Flynn JT. Hypertension in infancy: diagnosis, management and outcome. Pediatr Nephrol. 2012; 27(1): 17-32, doi: 10.1007/s00467-010-1755-z, indexed in Pubmed: 21258818.

9. Kimble KJ, Darnall RA, Yelderman M, et al. An automated oscillometric technique for estimating mean arterial pressure in critically ill newborns. Anesthesiology. 1981; 54(5): 423-425, indexed in Pubmed: 7224215

10. Tahir K, Mujeeb M, Khalid S. Management of Hypertensive nephropathy. J Biomed Sci and Res. 2010; 2: 295-301.

11. Zhu G, He F, Gu Y, et al. Angioplasty for pediatric renovascular hypertension: a 13-year experience. Diagn Interv Radiol. 2014; 20(3): 285292, doi: 10.5152/dir.2014.13208, indexed in Pubmed: 24675165.

12. Radanović B, Cacić Z, Perkov D, et al. Endovascular therapy of renovascular hypertension in children: single center analysis. Eur J Pediatr Surg. 2009; 19(3): 135-140, doi: 10.1055/s-00291202860, indexed in Pubmed: 19360546. 